\title{
Acute, fatal postoperative myocardial infarction after laparoscopic cholecystectomy in a cardiac patient
} -A case report-

\author{
Jae Jun Lee ${ }^{1}$, Sung Mi Hwang ${ }^{1}$, Hyoung Soo Kim² ${ }^{2}$ Byoung Yoon Ryu ${ }^{3}$, Jin Kim ${ }^{1}$, Ji Su Jang ${ }^{1}$, and \\ So Young $\operatorname{Lim}^{1}$
}

Departments of ${ }^{1}$ Anesthesiology and Pain Medicine, ${ }^{2}$ Thoracic and Cardiovascular Surgery, ${ }^{3}$ General Surgery, School of Medicine, Hallym University, Chuncheon, Korea

This report presents the case of a 63-year-old man who had a myocardial infarction leading to coronary artery bypass graft 2 years earlier who subsequently underwent elective laparoscopic cholecystectomy. After an uneventful operation, the patient developed an acute postoperative myocardial infarction in the recovery room and died 19 days postoperatively. Anesthesiologists should be aware of the rare possibility of acute, fatal postoperative myocardial infarction and consider this complication when they perform the preoperative risk evaluation, anesthesia, and postoperative care for cardiac patients undergoing noncardiac surgery. (Korean J Anesthesiol 2010; 59: S110-S113)

Key Words: Myocardial infarction, Noncardiac surgery.

Postoperative myocardial infarction (MI) after noncardiac surgery is one of the most serious and prognostically ominous complications of surgery and anesthesia. Reported rates of postoperative $\mathrm{MI}$ in patients undergoing noncardiac surgery vary widely. While the incidence of postoperative MI in the general noncardiac surgical population is relatively very low (less than 1\%) [1,2], the incidence is substantially higher in subgroups of patients with known cardiovascular risk factors and of older age [3].

We present a patient who underwent coronary artery bypass graft (CABG) 2 years earlier who had an acute postoperative MI in the recovery room after laparoscopic cholecystectomy.

\section{Case Report}

A 63-year-old man visited the emergency center of our hospital with epigastric pain and a fever. He was admitted for further evaluation and management. The diagnosis was gallbladder adenomyomatosis and he was scheduled for laparoscopic cholecystectomy (LC). He had hypertension and atrial fibrillation. He had experienced unstable angina, which led to stent insertion in the left anterior descending (LAD) coronary artery three times 12, 10, and 3 years earlier, respectively. Two years earlier, he underwent CABG due to MI. Coronary angiography performed just before the CABG showed

Received: March 30, 2010. Revised: April 15, 2010. Accepted: April 27, 2010.

Corresponding author: Sung Mi Hwang, M.D., Department of Anesthesiology and Pain Medicine, School of Medicine, Hallym University, Gyodong, Chuncheon 200-704, Korea. Tel: 82-33-240-5594, Fax: 82-33-252-0941, E-mail: h70sm@hallym.or.kr

(c) This is an open-access article distributed under the terms of the Creative Commons Attribution Non-Commercial License (http:// creativecommons.org/licenses/by-nc/3.0/), which permits unrestricted non-commercial use, distribution, and reproduction in any medium, provided the original work is properly cited. 
severe triple vessel disease. His medical regimen at the time of admission for elective LC included: bisoprolol, nicorandil, cilazapril, aspirin, isosorbide mononitrate, fluvastatin, and diltiazem.

The preoperative electrocardiogram (ECG) on this admission showed nonspecific ST-T changes (Fig. 1A) and coronary angiography performed after this admission showed total occlusion from the proximal to the distal parts of the circumflex artery with intracoronary collaterals, diffuse $30 \%$ stenosis of the proximal and middle parts of the LAD with stents, 95\% stenosis of the hypoplastic right coronary artery, and a patent left internal mammary artery to the obtuse marginal artery. The left ventricular function was preserved, with an ejection fraction of 0.59 . The chest X-ray was within normal limits. His preoperative AST, ALT, hemoglobin and hematocrit were $48 \mathrm{IU} /$ L, $87 \mathrm{IU} / \mathrm{L}, 13.2 \mathrm{~g} / \mathrm{dl}$ and $36.9 \%$. Other preoperative laboratory findings were within normal limits. He was premedicated with glycopyrrolate $0.2 \mathrm{mg}$ and midazolam $1.5 \mathrm{mg}$. The patient repeatedly refused a treadmill test.

In the operating room, monitoring included a five-lead ECG with continuous ST segment analysis, pulse oximetry, noninvasive blood pressure, end-tidal $\mathrm{CO}_{2}$, esophageal temperature, bispectral index, and an arterial catheter placed in the left radial artery. General anesthesia was induced uneventfully with a bolus of etomidate $21 \mathrm{mg}$ and rocuronium

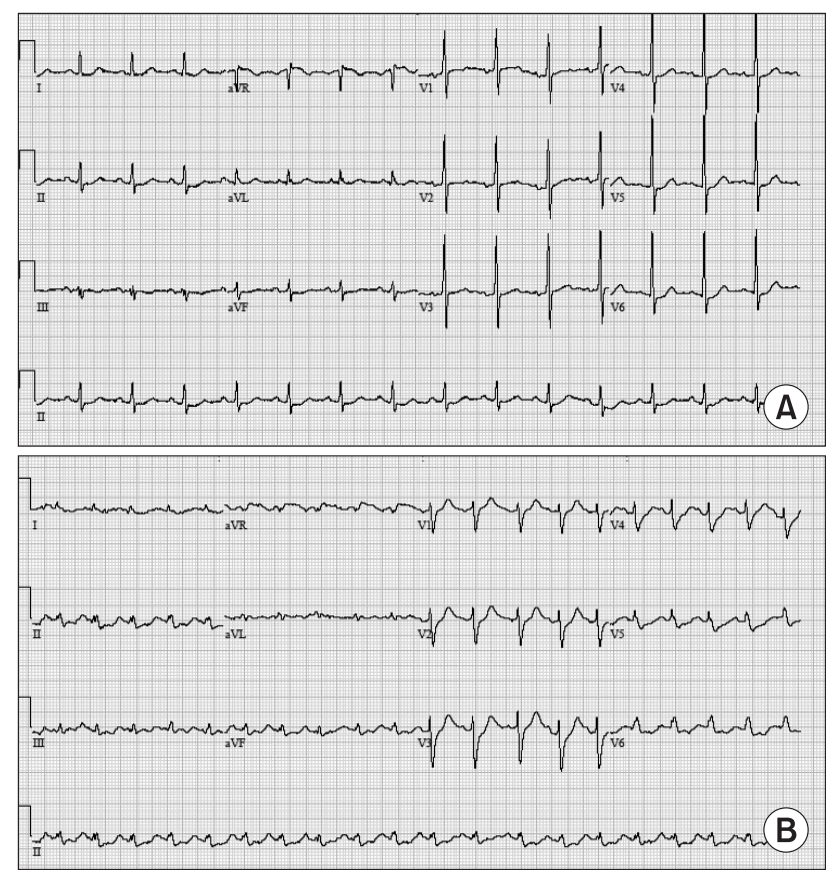

Fig. 1. (A) The preoperative electrocardiogram (ECG) shows nonspecific ST-T changes. (B) The six-lead portable ECG shows sinus tachycardia and ST-depression in leads II, III, aVF, V5, and V6 when the patient complained of chest pain in the recovery room.
$50 \mathrm{mg}$. Alfentanil $500 \mu \mathrm{g}$ was given at the time of intubation. Anesthesia was maintained with $2 \mathrm{~L} / \mathrm{min}$ oxygen, $2 \mathrm{~L} / \mathrm{min}$ nitrous oxide, and isoflurane with the concentration adjusted to maintain hemodynamic stability. Nitroglycerin (NTG) was given as a continuous intravenous dose of $0.5 \mu \mathrm{g} / \mathrm{kg} / \mathrm{min}$ for coronary vasodilation. The patient was ventilated by a semiclosed circular circuit with a tidal volume of $700 \mathrm{ml}$ and respiratory rate 12 breaths per minute. The blood pressure and pulse rate were stable throughout the operation at $130 / 80 \mathrm{mmHg}$ and 80 beats/ min, respectively. However, the ECG showed atrial fibrillation during the operation. Intraoperative intra-abdominal pressure (IAP) value of $13 \mathrm{mmHg}$ was achieved by insufflating the abdominal cavity with a carbon dioxide $\left(\mathrm{CO}_{2}\right)$. Intraoperative arterial blood gas analysis showed $\mathrm{pH}=7.37, \mathrm{PaCO}_{2}=39.1$ $\mathrm{mmHg}, \mathrm{PaO}_{2}=195 \mathrm{mmHg}, \mathrm{HCO}_{3}{ }^{-}=22 \mathrm{mEq} / \mathrm{dl}, \mathrm{Hb}=13.6 \mathrm{~g} /$ $\mathrm{dl}, \mathrm{Na}=135 \mathrm{mEq} / \mathrm{dl}$, and $\mathrm{K}^{+}=4.0 \mathrm{mEq} / \mathrm{dl}$. The operation took 1 $\mathrm{h}$ and $5 \mathrm{~min}$, and was uneventful. During surgery, he received $520 \mathrm{ml}$ of replacement fluid and the urine output was $80 \mathrm{ml}$. He was subsequently transferred to the recovery room for further observation. The initial blood pressure in the recovery room was $130 / 80 \mathrm{~mm} / \mathrm{Hg}$ and the three-lead ECG showed atrial fibrillation with 80 beats $/ \mathrm{min}$. In the recovery room, the patient still received the continuous NTG infusion that was started in the operating room.

Forty minutes after arriving at the recovery room, the patient complained of chest pain and dyspnea. His vital signs at that time were blood pressure $75 / 40 \mathrm{mmHg}$, heart rate 120 beats/ min, and $\mathrm{O}_{2}$ saturation $85 \%$. The ECG showed ST depression. He was intubated promptly; the NTG was stopped, and he was given an injection of phenylephrine $100 \mu \mathrm{g}$. The six-lead portable ECG (Fig. 1B) and cardiac markers were checked, and a central venous catheter was inserted in the left subclavian vein. The initial central venous pressure (CVP) value read $5 \mathrm{mmHg}$. We called a cardiologist and a chest surgeon. Soon afterward, the patient became asystolic on the ECG. Cardiopulmonary resuscitation (CPR) was begun immediately and epinephrine $1 \mathrm{mg}$ and atropine $0.5 \mathrm{mg}$ were administered initially and at 3-min intervals. Simultaneously, dopamine $20 \mu \mathrm{g} / \mathrm{min} / \mathrm{kg}$ was started. During CPR, a portable transthoracic echocardiogram was obtained, which showed hypokinesia of the inferior, lateral, and posterior walls and akinesia of the anterior wall. The estimated left ventricular ejection fraction was $10 \%$. The patient was transferred to the angiography room for insertion of an intra-aortic balloon pump (IABP). After the IABP was inserted, the patient's condition did not change. Immediately afterward, ECMO (Capiox Emergency Bypass System ${ }^{\circledR}$, TerumoInc., Tokyo, Japan) was begun at flow rates between 2.8 and $4 \mathrm{~L} / \mathrm{min}$. Serum troponin-I values increased form $0.04 \mu \mathrm{g} / \mathrm{L}$ at the time of acute event in the recovery room to $12.52 \mu \mathrm{g} / \mathrm{L} 3 \mathrm{~h}$ later the event (normal range $<0.05 \mu \mathrm{g} / \mathrm{L}$ ). The left ventricular ejection fraction 
improved to $25 \%$. Unfortunately, the patient died 19 days after surgery.

\section{Discussion}

Perioperative myocardial infarction is one of the most important predictors of short- and long-term morbidity and mortality associated with noncardiac surgery [4]. The American College of Cardiology and American Heart Association (ACC/ AHA) practice guidelines recommend assessing patients' comorbidities and exercise tolerance, as well as the type of surgery to be performed, to determine the overall risk of perioperative cardiac complications [5]. Based on this assessment, selected patients should undergo provocative cardiac testing; others may require interventions.

In our case, a patient who underwent CABG because of a MI 2 years earlier was undergoing an elective LC. According to the risk of various types of noncardiac surgical procedures stratified by the ACC/AHA practice guidelines, elective LC, which is intraperitoneal surgery, is a surgical procedure at intermediate cardiac risk [5]. The reported cardiac risk of intermediate cardiac risk surgical procedures is generally less than $5 \%$. However, during laparoscopic surgery, peritoneal insufflation to IAPs higher than $10 \mathrm{mmHg}$ induces significant alterations of hemodynamics [6]. These disturbances are characterized by decreases in cardiac output, increased arterial pressures, and elevation of systemic and pulmonary vascular resistance. Especially, the decrease in cardiac output is proportional to the increase in IAP [7]. Anesthesiologists should consider the hemodynamic changes associated with laparoscopic operation during preoperative surgical risk evaluation of cardiac patients. Concerning the cardiac risk with a previous CABG, if the patient has had complete surgical revascularization in the previous 5 years, and if his or her clinical status has remained stable without recurrent signs or symptoms of ischemia in the interim, the likelihood of perioperative cardiac death or MI is extremely low [8]. Our patient had no recurrent signs and had preserved left ventricular function with an ejection fraction of 0.59 , and was predicted to be at low cardiac risk because of the previous CABG.

The patient had hypertension and atrial fibrillation. Hypertension is an important risk factor for ischemic heart disease and results in increased systemic vascular resistance, decreased intravascular volume, and an exaggerated pressor response [9]. Furthermore, hypertension is one of the etiologies most frequently associated with atrial fibrillation (AF) [10], which can produce ischemia by increasing myocardial oxygen demand. In our case, the hypertension was well controlled during the preoperative and intraoperative periods, while the intraoperative and postoperative ECG showed AF.
Considering our preoperative evaluations, we regret that the patient's functional capacity was not checked with a treadmill test, although the patient refused the test to the last. Ergometric measurements on a treadmill inducing ischemia at low-level exercise $[<5$ metabolic equivalents (MET) or heart rate $<100 /$ $\mathrm{min}$ ] identify a high-risk group, whereas the achievement of more than 7 MET (or heart rate $>130 / \mathrm{min}$ ) without ischemia identifies a low-risk group [11].

Another missed point of this case was the use of phenylephrine immediately after development of the symptoms considering low blood pressure and rapid heart rate. Phenylephrine is a selective $\alpha_{1}$-agonist and commonly used when peripheral vasoconstriction is needed and cardiac output is adequate, as in patients with coronary artery disease to increase coronary perfusion pressure without chronotropic side effects [12]. However, ejection fraction of him was decreased with hypokinesia. Anesthesiologists should consider the cardiac output state before use of drug and we thought that norepinephrine might be better than phenylephrine in our case or similar case.

Most ischemic episodes tend to start at the end of surgery and during emergence from anesthesia [13]. This period is characterized by increases in sympathetic tone and procoagulant activity. Increased sympathetic tone can result in increases in arterial pressure, heart rate, and cardiac contractility, and these conditions might lead to subendocardial ischemia by increasing myocardial oxygen demand and eventual myocardial infarction. In addition, the intense procoagulant activity and sympathetic stimulation in the postoperative period have been implicated in the development of coronary vasospasm, thrombosis, and the rupture of atheromatous plaque, leading to myocardial ischemia and infarction [4]. However, this mechanism is not very plausible because ST-depression is the most common ECG change and the classic ST-elevation marker of acute coronary occlusion is rare in the postoperative period [14]. Furthermore, postoperative MI has always been considered a relatively late complication, with the peak occurrence on the third day after surgery, and with an exceptionally high mortality of $30-70 \%$ [15].

In our case, ischemic symptoms of chest pain and STdepression developed $40 \mathrm{~min}$ after surgery, while postoperative MI unusually occurs within $1 \mathrm{~h}$ after surgery in the recovery room. This case showed that postoperative MI can develop early after surgery, so anesthesiologists should cautiously monitor patients with coronary artery disease and cardiac problems in the recovery room. Other factors of concern for anesthesiologists-postoperative pain and physiological and emotional stress-may all combine to cause tachycardia, hypertension, increased cardiac output, and fluid shifts, which might result in subendothelial ischemia and eventual MI in 
high-risk patients [14]. Although patient controlled analgesia (PCA) was not applied routinely after LC in our hospital, in this case, PCA should be considered and might be helpful to the patient.

In conclusion, we described an acute, fatal postoperative MI in a cardiac patient with preserved left ventricular function after noncardiac surgery. Anesthesiologists must be aware that this rare complication could happen in cardiac patients after surgery. Anesthesiologists should always consider this complication when evaluating preoperative risk, performing anesthesia, and managing postoperative patient care, as well as the development of postoperative ischemic symptoms, including chest pain or ST-depression.

\section{References}

1. Selim M. Perioperative stroke. NEJM 2007; 356: 706-13.

2. Tarhan S, Moffitt EA, Taylor WF, Giuliani ER. Myocardial infartion after general anesthesia. JAMA 1972; 220: 1451-4.

3. Lee TH, Marcantonio ER, Mangione CM, Thomas EJ, Polanczyk CA, Cook EF, et al. Derivation and prospective validation of a simple index for prediction of cardiac risk of major non-cardiac surgery. Circulation 1999; 100: 1043-9.

4. Mangano DT. Perioperative cardiac morbidity. Anesthesiology 1990; 72: 153-84.

5. Eagle KA, Berger PB, Calkins H, Chaitman BR, Ewy GA, Fleischmann KE, et al. ACC/AHA guideline update for perioperative cardiovascular evaluation for noncardiac surgery-executive summary: a report of the American College of Cardiology/American Heart Association Task Force on Practice Guidelines (Committee to Update the 1996 Guidelines on Perioperative Cardiovascular Evaluation for Noncardiac Surgery). J Am Coll Cardiol 2002; 39: 542-53.
6. Struthers AD, Cuschieri A. Cardiovascular consequences of laparoscopic surgery. Lancet 1998; 352: 568-70.

7. Ivankovich AD, Miletich DJ, Albrecht RF, Heyman HJ, Bonnet RF. Cardiovascular effects of intraperitoneal insufflation with carbon dioxide insufflation and nitrous oxide in the dog. Anesthesiology 1975; 42: 281-7.

8. Mahar LJ, Steen PA, Tinker JH, Vlietstra RE, Smith HC, Pluth JR. Perioperative myocardial infarction in patients with coronary artery disease with and without aorta-coronary artery bypass grafts. J Thorac Cardiovasc Surg 1978; 76: 533-7.

9. Fleisher LA, Barash PG. Preoperative evaluation of the cardiac patient for noncardiac surgery. Yale J Biol Med 1993; 66: 385-95.

10. Mackstaller LL, Alpert JS. Atrial fibrillation: a review of mechanism, etiology, and therapy. Clin Cardiol 1997; 20: 640-50.

11. Weiner DA, Ryan TJ, McCabe CH, Chaitman BR, Sheffield LT, Freguson JC, et al. Prognostic importance of a clinical profile and exercise test in medically treated patients with coronary artery disease. J Am Coll Cardiol 1984; 3: 772-9.

12. Glick DB. The Autonomic Nervous system. In: Miller's Anesthesia. 7th ed. Edited by Miller RD, Eriksson LI, Fleisher LA, WienerKronish JP, Young WL: Philadelphia, Elsevier Publishers. 2009, pp 261-304.

13. Landesberg G, Mosseri M, Zahger D, Wolf Y, Perouansky M, Anner $\mathrm{H}$, et al. Myocardial infarction after vascular surgery: the role of prolonged stress-induced, ST-depression type ischemia. J Am Coll Cardiol 2001; 37: 1839-45.

14. Landesberg G, Luria MH, Cotev S, Eidelman LA, Anner H, Mosseri $\mathrm{M}$, et al. Importance of long-duration postoperative ST-segment depression in cardiac morbidity after vascular surgery. Lancet 1993; 341: 715-9.

15. Goldman L, Adler J. General anesthesia and non-cardiac surgery in patients with heart disease. In: Heart disease: a textbook of cardiovascular medicine. 6th ed. Edited by Braunwald E, Zipes D, Libby P: Philadelphia, WB Saunders Publishers. 2001, pp 2084-97. 